\section{Distribuição espaço-temporal dos candidatos à doação de sangue da Fundação Hemominas, Belo Horizonte, Minas Gerais, Brasil, nos anos de 1994 e 2004}

\author{
Spatial-temporal distribution of potential blood \\ donors at the Hemominas Foundation, \\ Belo Horizonte, Minas Gerais State, Brazil, \\ in 1994 and 2004
}

\begin{abstract}
The main goal of this study was to describe the spatial and temporal distribution of candidates for blood donation in Belo Horizonte, Minas Gerais State, Brazil, who appeared at the Hemominas Foundation in 1994 and 2004. The study also compared the candidates for age, gender, and clinical approval for blood donation in space and space-time. Data were obtained from a cross-sectional study for 1994 and were randomly selected from all donor candidates for 2004. The samples were georeferenced using the residential address. The spatial analysis techniques employed were: Kernel maps, thematic maps of Bayesian empirical rates and crude rates, and Moran Global. According to the findings, spatial distribution of candidates was non-random. The Kernel maps helped detect points with higher or lower concentration of candidates. Thematic maps described the concentration of candidates in relation to the population for the various categories. The results could help detect areas for actions targeting donor recruitment and areas with specific blood donation public campaign needs.
\end{abstract}

Blood Donors; Spatial Analysis; Hemotherapy Service

\author{
Camila Caram 1 \\ Mônica Silva Monteiro-de-Castro 2 \\ Waleska Teixeira Caiaffa 1 \\ Claudia Di Lorenzo Oliveira ${ }^{3}$ \\ Anna Bárbara de Freitas Carneiro Proietti 3 \\ Maria Cristina de Mattos Almeida 2 \\ Stela Brener 3 \\ Fernando Augusto Proietti 1
}

\section{Introdução}

Várias condições clínicas estão associadas à prescrição e indicação de transfusões de sangue e produtos derivados do sangue. Destacam-se, por exemplo, cirurgias eletivas ou de urgência e patologias como hemofilia, câncer e queimaduras (Fundação Centro de Hematologia e Hemoterapia de Minas Gerais. http://www.hemominas. mg.gov.br, acessado em 23/Set/2004). De acordo com a Constituição Federal de 1998, todo o sangue utilizado em transfusões deve ser obtido através de doações voluntárias.

A falta de doadores e elevados índices de inaptidão clínica e sorológica podem resultar em déficit nos estoques de sangue, gerando conseqüências adversas para os indivíduos e a saúde coletiva. Portanto, é de extrema importância que se estimule de várias formas a doação de sangue, seja pela fidelização dos doadores ou mobilização permanente da população.

No Brasil, em 2004, aproximadamente 1,7\% dos brasileiros doaram sangue 1 . Segundo a Organização Mundial da Saúde (OMS), se pelo menos 3\% doarem sangue as necessidades de um país serão atendidas 1 . Levantamento feito pela Coordenação da Política Nacional de Sangue do Ministério da Saúde constatou que, nos primeiros meses de 2004, houve redução média de $15 \%$ nos estoques de sangue em 12 dos 27 estados brasileiros ${ }^{1}$. 
De todas as coletas de sangue do Brasil em 2002, 8,5\% (259.261) foram realizadas em Minas Gerais (Agência Nacional de Vigilância Sanitária. http://www.anvisa.gov.br/sangue/index.htm, acessado em 23/Set/2004). Em 2006, o número de tentativas de doação no Estado de Minas Gerais cresceu para 335 mil, sendo que $66 \%$ eram do sexo masculino e $77 \%$ tinham menos de 40 anos 2. Este perfil é diferente do relatado em estudo realizado em 2000, nos Estados Unidos, que relata $50 \%$ dos doadores do sexo feminino, $71 \%$ de candidatos de repetição e $44 \%$ dos candidatos com idade entre 16 e 39 anos 3 .

A coleta e transfusão do sangue no Brasil estão sob a regulamentação da Agência Nacional de Vigilância Sanitária (ANVISA) e é estruturada a partir de 26 hemocentros coordenadores, entre eles a Fundação Hemominas. Esta possui 21 unidades, sendo quatro unidades localizadas na capital (Belo Horizonte) e 17 no interior do estado. Das quatro unidades localizadas na capital apenas duas são unidades de coleta de sangue: o Hemocentro de Belo Horizonte, maior unidade de coleta do estado, localizado na região ao centro de Belo Horizonte e responsável por aproximadamente $90 \%$ das coletas de sangue do município; e o Hospital Júlia Kubitschek, localizado no extremo sul de Belo Horizonte e responsável por $10 \%$ das coletas (Fundação Centro de Hematologia e Hemoterapia de Minas Gerais; http://www.hemominas.mg.gov.br, acessado em 23/Set/2004).

O estudo de fatores relacionados à decisão de doar sangue, bem como as características sóciodemográficas dos doadores e/ou relacionadas à aptidão clínica dos mesmos, apesar de pouco explorado em nosso país, é de fundamental importância para o planejamento de campanhas para a captação dos doadores e sua fidelização ${ }^{4}$.

As características físicas e sociais do espaço geográfico onde as pessoas vivem estão associadas à ocorrência de diversos eventos relacionados à saúde 5 . Apesar da tendência crescente do uso de mapas e de análise espacial em epidemiologia, pouco tem sido estudado em relação à distribuição espacial de candidatos à doação de sangue.

Neste cenário, o presente estudo teve como objetivo descrever e comparar no espaço e no tempo, a distribuição de candidatos à doação de sangue do Hemocentro de Belo Horizonte, nos anos de 1994 e 2004, levando-se em conta as características demográficas (sexo e idade), epidemiológicas e comportamentais da doação, avaliadas através do indicador de aptidão clínica para doação de sangue e, considerando ainda, a população residente em cada região.

\section{Métodos}

\section{Local do estudo}

O Município de Belo Horizonte, capital do Estado de Minas Gerais, possui população de 2.238.332 habitantes, distribuída por uma área de cerca de $330 \mathrm{~km}^{2}$. Dos 2,2 milhões de habitantes do município, 64\% (1,4 milhões) têm entre 18 e 64 anos correspondente à faixa etária permitida para a doação de sangue (Instituto Brasileiro de Geografia e Estatística. Censo demográfico 2000. http://www.ibge.gov.br).

\section{Fonte de dados e critérios de inclusão}

$\mathrm{O}$ candidato à doação de sangue foi caracterizado como "indivíduo que compareceu ao $\mathrm{HBH}$ com a intenção de fazer uma doação de sangue" 4 (p. 109).

A amostra de 1994 foi composta por candidatos à doação de sangue do Hemocentro de Belo Horizonte, residentes no Município de Belo Horizonte, amostrados através do estudo transversal denominado Aspectos Sócio-econômicos, Demográficos e Culturais da Doação de Sangue em Minas Gerais, conduzido em parceria entre a Fundação Hemominas e a Universidade Federal de Minas Gerais (UFMG) e financiado pela Fundação de Amparo à Pesquisa de Minas Gerais (FAPEMIG) 4,6. As informações foram obtidas por meio de entrevistas face a face. Conforme metodologia estabelecida, testada e avaliada no estudo piloto, os candidatos à doação eram abordados de forma consecutiva após cadastramento e antes da triagem clínica, quando eram convidados a participar do estudo. Dados relativos a sexo e idade foram confrontados entre a amostra e o sistema de informação do Hemocentro de Belo Horizonte, não sendo verificada diferença estatisticamente significante ${ }^{4}$.

Para comparação com a amostra de 1994 foram sorteados, na razão de 1:1, 1.873 candidatos à doação de sangue do ano de 2004 registrados no banco de dados do cadastro realizado antes da avaliação clínica do Hemocentro de Belo Horizonte. Para este sorteio foram considerados todos os candidatos que se apresentaram com intenção de doar sangue em 2004 e que residiam no Município de Belo Horizonte, sendo a verificação do município de residência realizada através do código de endereçamento postal (CEP).

Os candidatos amostrados foram georreferenciados de acordo com o endereço exato de residência. Caso o endereço não constasse na base geográfica, optou-se por georreferenciá-lo ao número mais próximo pertencente ao mesmo logradouro. 


\section{Análise dos dados}

Os candidatos à doação foram classificados de acordo com as seguintes variáveis: sexo (masculino e feminino), aptidão clínica (aptos e inaptos) e idade (menor ou igual a 29 anos e maior de 29 anos). Para assegurar representatividade destas categorias, os candidatos que não puderam ser georreferenciados foram comparados aos georreferenciados através do teste $\chi^{2} \mathrm{de}$ Pearson.

Com o objetivo de se explorar o comportamento espacial dos eventos, foram gerados mapas de superfície (mapas de Kernel) 7,8, dos anos estudados e das categorias de interesse. Para isso cada evento (candidato a doação de sangue) foi relacionado a um ponto no mapa, aos quais estavam associadas as categorias de interesse. Os parâmetros adotados para a construção dos mapas de superfície foram: algoritmo quadrático Kernel baseado na densidade de pontos e raio adaptativo. A grade utilizada foi de 250 colunas, o que corresponde, para o Município de Belo Horizonte, à aproximadamente 85 metros. Esta análise permitiu a detecção de áreas com maior e menor intensidade de eventos além de permitir identificar áreas de transição entre elas.

Complementarmente, para avaliar a chance de um indivíduo se candidatar para doação de sangue foram calculadas as taxas de candidatos à doação por área de abrangência. As áreas de abrangência são definidas como os territórios de cobertura dos centros de saúde e constituídas de setores censitários contíguos. Ressalta-se que as áreas de abrangência têm características heterogêneas entre si quanto às condições sócio-econômicas, refletindo a heterogeneidade dos setores censitários do município que compõem cada uma das áreas de abrangência (Prefeitura $\mathrm{Mu}$ nicipal de Belo Horizonte. http://www.pbh.gov. $\mathrm{br} / \mathrm{smsa} / \mathrm{montapagina.php?pagina=instituicao.}$ html, acessado em 14/Nov/2005).

No cálculo das taxas como numerador utilizou-se a contagem dos eventos (candidatos à doação) dentro de cada área de abrangência. Como denominador utilizou-se a população desta mesma área de abrangência obtida no Censo Demográfico de 2000 com as mesmas características dos eventos. Assim, o denominador para os candidatos foi a totalidade de indivíduos residentes na região com idade entre 18 e 64 anos; para aqueles do sexo masculino foi a população entre 18 e 64 anos do sexo masculino; para as mulheres foi a população entre 18 e 64 anos do sexo feminino. Em se tratando da categoria de idade até 29 anos utilizou-se como denominador a população residente na região com idade entre 18 e 29 anos; e, para aqueles acima de 29 anos utilizou-se como denominador a população entre 30 e 64 anos.

Para aptos e inaptos, por não existirem estimativas da população real candidata, nem estimativas de aptidão clínica em cada região, utilizou-se como denominador toda a população residente na área de abrangência com idade entre 18 e 64 anos. As taxas calculadas foram denominadas taxas brutas por não apresentarem nenhum tipo de correção, ou seja, referem-se às estimativas de candidatos pela população.

A partir das taxas brutas foram geradas as taxas Bayesianas empíricas 7,8,9, estimador local, de cada uma das áreas de abrangência dos 137 centros de saúde do município. Este método foi adotado por utilizar as informações das demais áreas que compõem a região de estudo para suavizar o valor da taxa bruta em cada área, diminuindo assim o efeito de flutuações aleatórias dos estimadores ${ }^{9}$. A correção Bayesiana é recomendada em situações onde as regiões possuem pequenas populações de risco, o que acarreta estimativas de risco muito instáveis 9 . E também em situações de eventos raros, onde o aumento absoluto de apenas um evento altera sensivelmente o valor da taxa bruta ${ }^{9}$. Esta situação se aplica às áreas de abrangência, pois (i) são áreas territoriais pequenas, algumas com população pequena e (ii) o número de eventos variou entre 0 e 44 (entre toda as classes), sendo a classe mais crítica a de candidatas em 1994 com o valor máximo de eventos (numerador) entre as áreas igual a 15.

Uma vez calculadas as taxas brutas e taxas Bayesianas empíricas, que foram multiplicadas por mil, foi verificada a existência de dependência espacial, ou seja, se os eventos estavam correlacionados no espaço. Para isso, utilizou-se o índice de Moran global, que testa a hipótese nula de independência espacial. Valores positivos e significativos (valor de $\mathrm{p} \leq 0,05$ ) do índice indicam autocorrelação direta, e valores negativos, autocorrelação inversa. Caso houvesse significância concluía-se que as taxas de candidatos à doação de sangue apresentaram estrutura espacial.

A partir das taxas brutas e das taxas Bayesianas empíricas foram gerados mapas temáticos onde a gradação de cor foi padronizada para os quartis do ano de 2004 para cada categoria.

Todas as análises foram realizadas nos programas MapInfo (MapInfo Corp., New York, Estados Unidos), Terraview (Instituto Nacional de Pesquisas Espaciais; http://www.dpi.inpe.br/ter raview), R (The R Foundation for Statistical Computing, Viena, Áustria; http:/ / www.r-project.org) e seu módulo SPLANCS 10.

Este estudo foi aprovado pelo colegiado de pós-graduação da Faculdade de Medicina da Universidade Federal de Minas Gerais e subme- 
tido ao comitê de ética em pesquisa da Fundação Hemominas (CEP nº. 129/05).

\section{Resultados}

Dos 3.527 amostrados no estudo de 1994 foram excluídos 1.383 indivíduos não residentes do Município de Belo Horizonte. Entre os 2.144 residentes no município, 271 (12,6\%) não foram georreferenciados por apresentarem inconsistência na declaração do endereço de residência. A amostra final do ano de 1994 foi composta por 1.873 candidatos.

O banco de dados de 2004 possuía 51.653 indivíduos, correspondente a todos os candidatos à doação que se apresentaram ao Hemocentro de Belo Horizonte ao longo do ano. Dos 35.834 candidatos residentes no Município de Belo Horizonte, foram sorteados, 1.873 para este estudo (margem de erro de $2,2 \%$ ).

A Tabela 1 apresenta a distribuição de freqüência das amostras de 1994 e 2004, de acordo com as categorias de interesse. Os testes $\chi^{2} \mathrm{de}$ Pearson referem-se às comparações das amostras finais com os candidatos residentes do $\mathrm{Mu}$ nicípio de Belo Horizonte. Todos os valores de p observados foram superiores a 0,05 caracterizando assim semelhança das amostras finais com os bancos de dados dos quais estas amostras foram originadas. Ou seja, as perdas relacionadas ao georreferenciamento foram homogêneas nas categorias de interesse.

A Figura 1 apresenta os mapas de Kernel para candidatos à doação em 1994 e 2004. Observa-se que em ambos os anos existiu maior concentração de candidatos na região ao centro do muni- cípio, sendo esta concentração visualmente mais acentuada no ano de 1994 do que em 2004. Os principais resultados observados nos mapas de Kernel para as categorias de interesse serão descritos a seguir (mapas não apresentados).

A diminuição da concentração de candidatos na região central entre 1994 e 2004 ocorreu também para todas as categorias estudadas (sexo, idade e aptidão clínica). Entretanto para mulheres, inaptos e maiores de 29 anos, este agrupamento pareceu reduzir mais acentuadamente do que para as categorias de comparação (homens, aptos e menores de 29 anos). Por outro lado, observou-se que neste intervalo de dez anos, a concentração na região ao norte do município tendeu a crescer, principalmente para mulheres, aptos e menores de 29 anos. Essas categorias apresentavam pequena concentração de eventos na região em 1994 e tenderam a apresentar maior concentração em 2004.

Já os homens concentravam-se mais no noroeste do município nos dois anos estudados. Nesta região, a concentração de mulheres só foi observada em 2004, e em 1994, também foi observada uma concentração de inaptos, que em 2004 apresentaram inversão do padrão de aptidão, agora com concentração de aptos. Esta inversão também pode ser observada na mesma região para as categorias de idade, enquanto em 1994, observou-se concentração um pouco mais acentuada de maiores de 29 anos, em 2004, esta se acentuou entre menores de 29 anos.

A Tabela 2 apresenta as médias, desvios-padrão, índice de Moran global e teste de significância das taxas brutas e das taxas Bayesianas empíricas. Por terem sido observados apenas valores positivos do índice de Moran global e va-

Distribuição de freqüência de candidatos e amostra georrefenciada de candidatos à doação de sangue do Hemocentro de Belo Horizonte (Fundação Hemominas), Minas Gerais, Brasil, nos anos de 1994 e 2004.

\begin{tabular}{|c|c|c|c|c|c|c|c|c|c|c|}
\hline & \multirow[t]{2}{*}{$\mathbf{n}$} & \multicolumn{3}{|c|}{ Sexo } & \multicolumn{3}{|c|}{ Aptidão clínica } & \multicolumn{3}{|c|}{ Idade (anos) } \\
\hline & & $\begin{array}{c}\text { Masculino } \\
\text { (\%) }\end{array}$ & $\begin{array}{c}\text { Feminino } \\
\text { (\%) }\end{array}$ & Valor de $p$ * & $\begin{array}{l}\text { Apto } \\
(\%)\end{array}$ & $\begin{array}{c}\text { Inapto } \\
\text { (\%) }\end{array}$ & Valor de $p$ & $\begin{array}{l}\leq 29 \\
(\%)\end{array}$ & $\begin{array}{c}>29 \\
(\%)\end{array}$ & Valor de $p$ * \\
\hline 1994 & & & & 0,930 & & & 0,837 & & & 0,749 \\
\hline Candidatos de Belo Horizonte & 2.144 & 75,9 & 24,1 & & 56,4 & 43,6 & & 60,0 & 40,1 & \\
\hline Georreferenciados & 1.873 & 75,8 & 24,2 & & 56,1 & 43,9 & & 60,4 & 39,6 & \\
\hline 2004 & & & & 0,255 & & & 0,904 & & & 0,514 \\
\hline Candidatos de Belo Horizonte & 35.834 & 64,0 & 36,0 & & 58,7 & 41,3 & & 66,2 & 33,8 & \\
\hline Georreferenciados & 1.873 & 62,7 & 37,3 & & 58,6 & 41,4 & & 65,5 & 34,5 & \\
\hline
\end{tabular}

* Valores de p referentes aos testes $\chi^{2}$ de Pearson. 
Mapas de Kernel dos candidatos à doação de sangue do Hemocentro de Belo Horizonte (Fundação Hemominas), Minas Gerais, Brasil, nos anos de 1994 e 2004
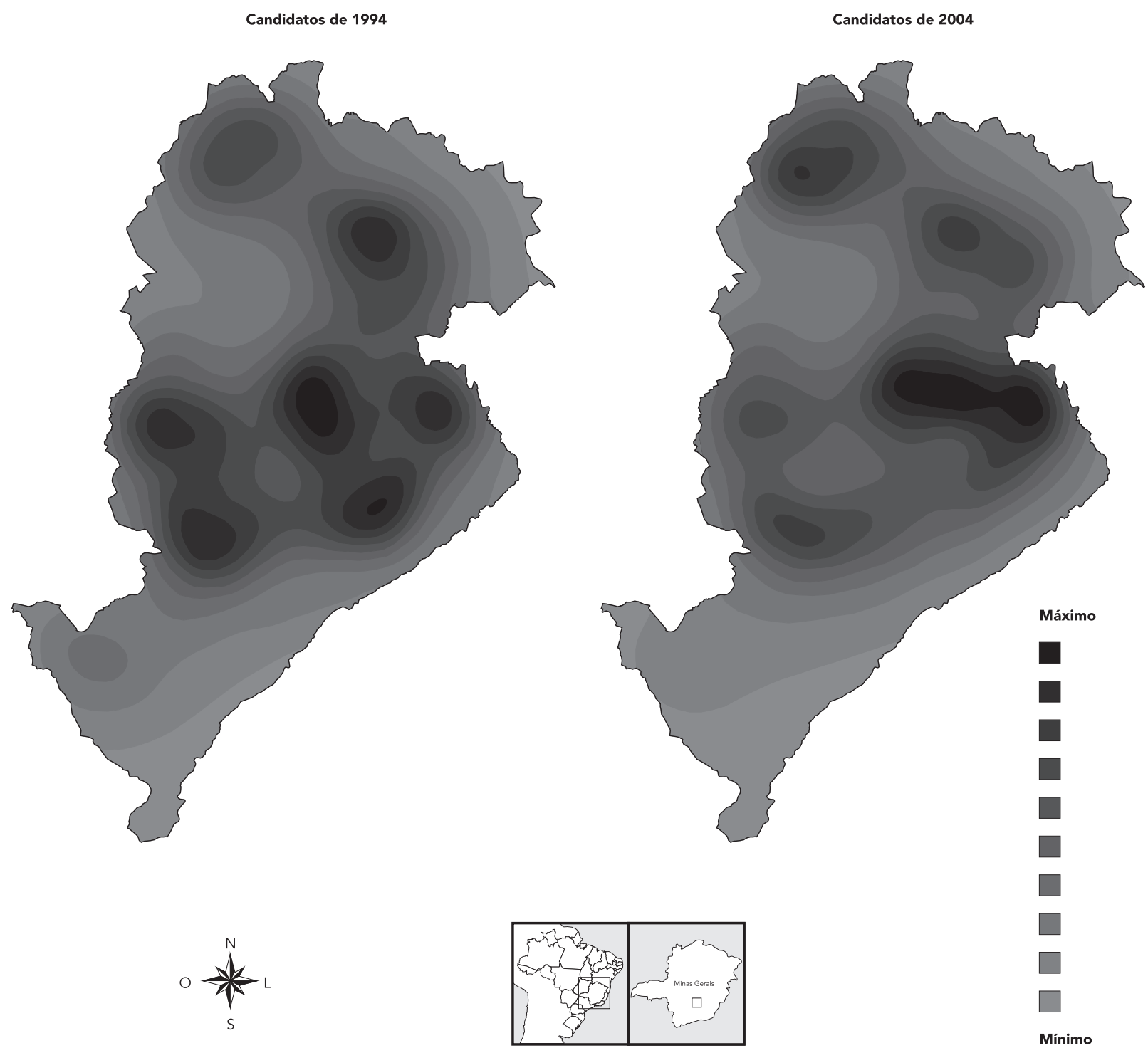

lores de p significativos $(\leq 0,05)$ conclui-se que os candidatos à doação tanto em 1994 quanto em 2004, de todas as categorias de interesse, apresentaram estrutura espacial e estavam correlacionados positivamente no espaço.

Os mapas na Figura 2 apresentam as taxas Bayesianas empíricas e as taxas brutas dos candidatos à doação para cada ano. Serão descritos os principais resultados para as taxas Bayesianas empíricas e também os resultados dos mapas temáticos das taxas Bayesianas empíricas das categorias estudadas (mapas não apresentados).

No ano de 1994, mulheres, idade $\leq 29$ anos e aptos apresentam áreas de abrangência com baixas taxas Bayesianas empíricas ao longo de todo município. Áreas de abrangência localizadas ao sul apresentavam baixas taxas Bayesianas empíricas em todas as categorias estudadas. Ho- 
Média, desvio-padrão (DP), índice de Moran global e teste de significância das taxas brutas e das taxas bayesianas empíricas de candidatos e categorias de interesse de candidatos à doação de sangue do Hemocentro de Belo Horizonte (Fundação Hemominas), Minas Gerais, Brasil, nas áreas de abrangência nos anos de 1994 e 2004.

\begin{tabular}{|c|c|c|c|c|c|c|c|c|}
\hline \multirow[t]{2}{*}{ Categoria } & \multicolumn{4}{|c|}{ Taxa Bayesiana empírica } & \multicolumn{4}{|c|}{ Taxa bruta } \\
\hline & Média & DP & $\begin{array}{c}\text { Índice de } \\
\text { Moran } \\
\text { global }\end{array}$ & Valor de $p$ * & Média & $\mathrm{DP}$ & $\begin{array}{c}\text { Índice de } \\
\text { Moran } \\
\text { global }\end{array}$ & Valor de $p$ * \\
\hline \multicolumn{9}{|l|}{ Sexo } \\
\hline Masculino & 2,131 & 0,517 & 0,601 & 0,001 & 2,153 & 0,926 & 0,143 & 0,006 \\
\hline Feminino & 0,586 & 0,233 & 0,539 & 0,001 & 0,607 & 0,446 & 0,170 & 0,006 \\
\hline \multicolumn{9}{|l|}{ Aptidão clínica } \\
\hline Aptos & 0,728 & 0,220 & 0,462 & 0,001 & 0,746 & 0,397 & 0,112 & 0,029 \\
\hline Inaptos & 0,585 & 0,159 & 0,699 & 0,001 & 0,591 & 0,300 & 0,160 & 0,005 \\
\hline \multicolumn{9}{|l|}{ Idade (anos) } \\
\hline$\leq 29$ & 2,149 & 0,536 & 0,619 & 0,001 & 2,165 & 1,034 & 0,174 & 0,005 \\
\hline$>29$ & 0,829 & 0,220 & 0,631 & 0,001 & 0,849 & 0,416 & 0,159 & 0,009 \\
\hline Candidatos de 2004 & 1,319 & 0,447 & 0,775 & 0,001 & 1,362 & 0,602 & 0,422 & 0,001 \\
\hline \multicolumn{9}{|l|}{ Sexo } \\
\hline Masculino & 1,771 & 0,632 & 0,734 & 0,001 & 1,812 & 0,943 & 0,271 & 0,001 \\
\hline Feminino & 0,919 & 0,328 & 0,743 & 0,001 & 0,956 & 0,554 & 0,249 & 0,001 \\
\hline \multicolumn{9}{|l|}{ Aptidão clínica } \\
\hline Aptos & 0,782 & 0,273 & 0,707 & 0,001 & 0,803 & 0,406 & 0,301 & 0,001 \\
\hline Inaptos & 0,541 & 0,201 & 0,787 & 0,001 & 0,559 & 0,323 & 0,261 & 0,001 \\
\hline \multicolumn{9}{|l|}{ Idade (anos) } \\
\hline$\leq 29$ & 2,340 & 0,745 & 0,804 & 0,001 & 2,350 & 1,095 & 0,322 & 0,001 \\
\hline$>29$ & 0,715 & 0,306 & 0,633 & 0,001 & 0,758 & 0,497 & 0,209 & 0,003 \\
\hline
\end{tabular}

* Valores de p referentes às permutações dos testes de pseudo-significância.

mens e maiores de 29 anos apresentaram áreas de abrangência com taxas Bayesianas empíricas elevadas em regiões dispersas do município. A categoria inaptos apresentou área de abrangência com valores elevados nos extremos norte, leste e oeste do município.

No ano de 2004, para todas as categorias estudadas, as áreas de abrangência mais ao norte do município apresentaram maiores taxas Bayesianas empíricas do que aquelas da região mais ao sul, com um claro crescimento dos valores das taxas Bayesianas empíricas no sentido sul-norte. Em outras palavras, quanto mais ao sul, menores foram as taxas Bayesianas empíricas.

Comparando-se os mapas de 1994 e 2004 observava-se que ao norte do município houve crescimento das taxas Bayesianas empíricas em quase todas as categorias, exceção para a categoria $>29$ anos, onde, de maneira geral, houve decréscimo, apesar das taxas manterem-se altas, com os maiores valores concentrados no extremo norte. Destaca-se também a região mais ao nordeste, com as maiores taxas Bayesianas empíricas do município em 2004 além das categorias sexo feminino, aptos e $\leq 29$ anos com as menores taxas Bayesianas empíricas em 1994 e valores tão altos quanto as outras categorias em 2004.

Em 1994, a região mais ao sul apresentava área de abrangência com baixas taxas Bayesianas empíricas de candidatos à doação de sangue independente do sexo, idade e aptidão. Este padrão manteve-se quando os mapas foram comparados aos do ano de 2004. Entretanto, destacam-se as categorias sexo feminino, aptos e maiores de 29 anos, onde, em 2004 algumas áreas de abrangência localizadas no sudeste do município apresentaram maiores taxas Bayesianas empíricas.

A região central apresentou área de abrangência com valores das taxas Bayesianas empíricas maiores em 2004 do que em 1994 em todas as 


\section{Figura 2}

Mapas temáticos das taxas Bayesianas empíricas e brutas de candidatos à doação de sangue do Hemocentro de Belo Horizonte (Fundação Hemominas) nas 137 áreas de abrangência dos centros de saúde do Município de Belo Horizonte, Minas Gerais, Brasil, nos anos de 1994 e 2004.

Taxa Bayesiana empírica
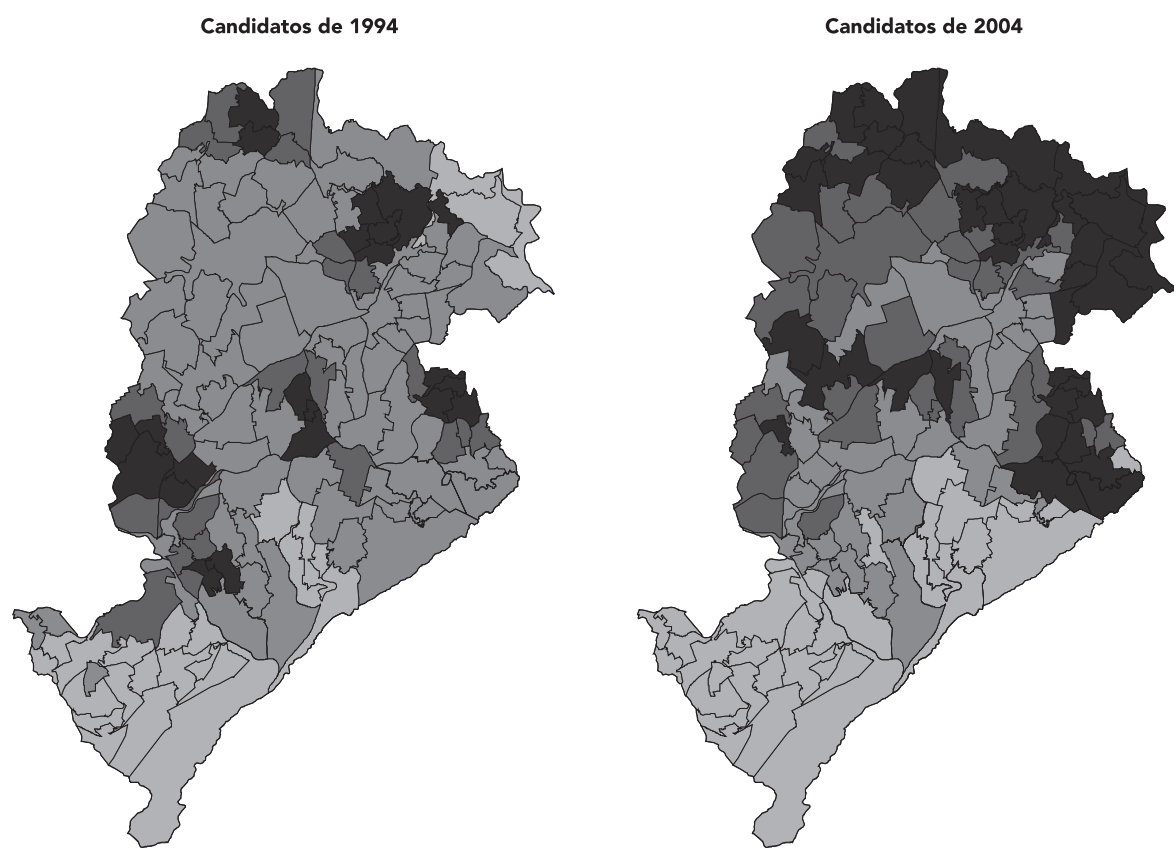

Taxa bruta
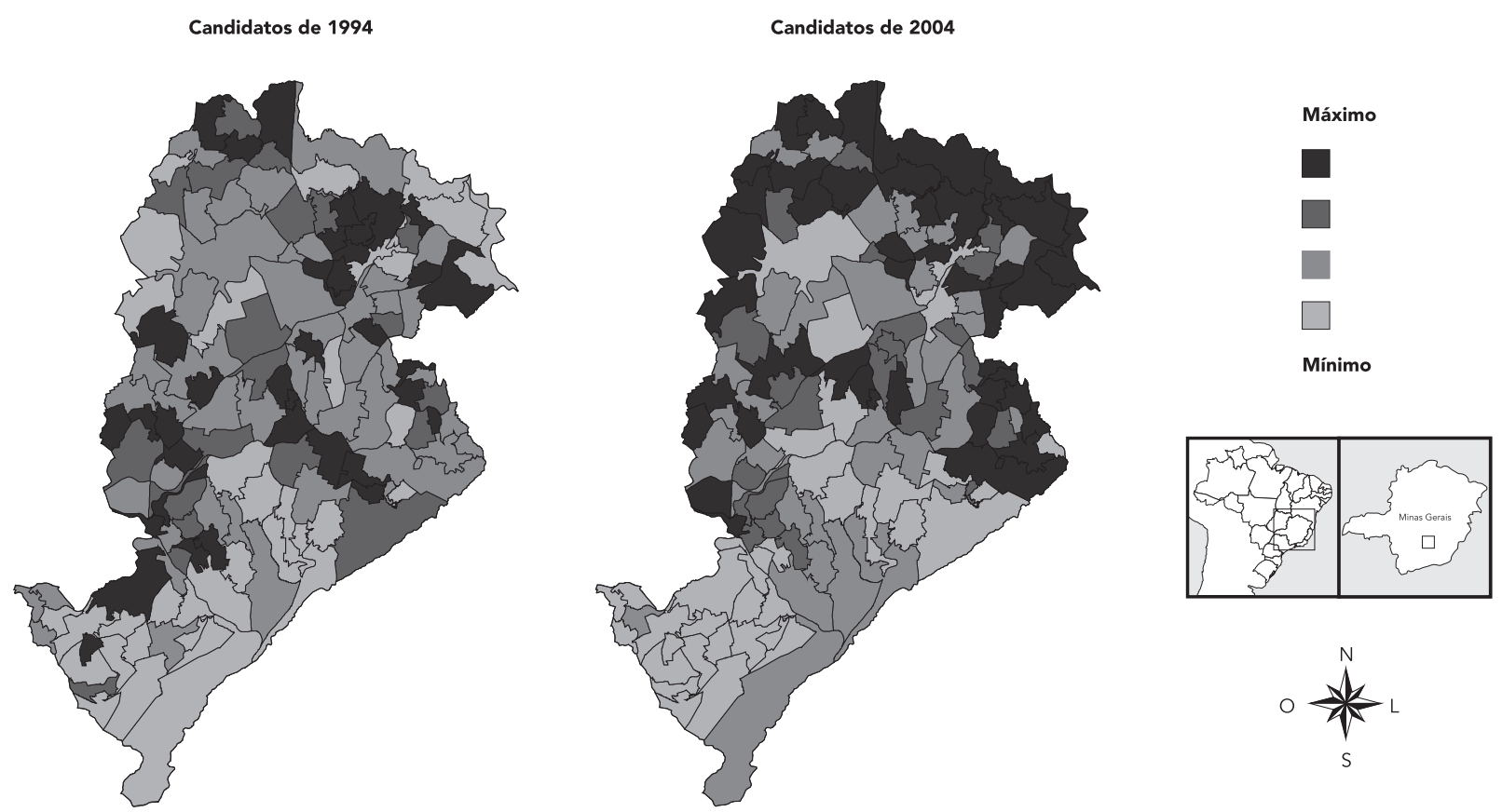
categorias, com destaque para a categoria sexo, onde mulheres apresentaram crescimento acentuado e homens apresentaram decréscimo das taxas Bayesianas empíricas.

\section{Discussão e conclusão}

Os resultados indicam que a distribuição espacial de candidatos à doação de sangue no $\mathrm{Mu}$ nicípio de Belo Horizonte, nos dois anos estudados, não foi aleatória, apresentando agrupamentos espaciais.

Foram observadas particularidades na concentração dos candidatos a doação nos anos estudados de acordo com sexo, idade e aptidão clínica. Também foram observados estrutura espacial e comportamentos heterogêneos para a unidade geográfica de análise utilizada (área de abrangência) quanto ao sexo, idade, aptidão clínica e ano calendário, permitindo algumas considerações no entendimento da dinâmica do processo de doação de sangue e auxiliando a elaboração de políticas e programas de saúde.

Ao se estudar o padrão espacial de candidatos à doação de sangue propõe-se uma nova perspectiva de monitoramento e ação. Em especial, ao se estudar o padrão de pontos pode-se objetivar estratégias de captação de doadores de sangue de caráter emergencial, visando repor, a curto prazo, os estoques de sangue quando estes são considerados baixos, através da detecção dos locais de concentração de potenciais candidatos à doação. E ao se estudar o padrão de áreas propõe-se a detecção de unidades geográficas onde os trabalhos de captação de doadores devam ser focados, no intuito de aumentar a quantidade relativa de candidatos.

O uso de mapas e da análise espacial no entendimento dos eventos relacionados à saúde tem sido observado em muitos estudos no campo da saúde pública. Muitos destes estudos trabalham com eventos na forma de processos pontuais $11,12,13,14,15$, outros analisam os eventos na forma de taxas 16,17,18,19,20,21. O uso dessas técnicas permite um melhor entendimento da dinâmica dos eventos relacionados à saúde no espaço, auxiliando na elaboração de políticas e programas de saúde.

Em estudo de 2004, Moran et al. 16 utilizaram taxas padronizadas de doadores de sangue no Reino Unido como medida do capital social para pequenas áreas. Os autores incluíram o espaço na modelagem multi-nível e correlacionaram essas taxas com variáveis epidemiológicas e sociais. Embora o estudo não apresente uma análise espacial com o uso de técnicas de análise especificas ou mapas, ressalta a importância de se considerar características locais, como o espaço físico, para o entendimento das motivações que levam à doação de sangue.

Monteiro-de-Castro et al. 11 estudaram a distribuição espacial da infecção pelo HTLV-I/II, em doadores de sangue da Fundação Hemominas, residentes em Belo Horizonte, de 1994 a 1996. Não encontraram evidências, através da diferença entre as funções $K$, de que os grupos estudados (doadores infectados e não infectados pelo HTLV-I/II) apresentassem distribuição espacial heterogênea quanto ao local residência.

A análise de pontos, apesar de muito útil, não considera a densidade populacional na região estudada, o que pode levar a inferências equivocadas quanto a real concentração de eventos. Em outras palavras, uma vez identificada uma área de concentração de pontos, devemos questionar se essa concentração não seria apenas um reflexo da concentração da população na região onde foi detectada esta concentração, ou ainda, se a baixa concentração de eventos em determinada região não seria apenas o reflexo da - quase - ausência de população.

Para verificar se áreas com maior concentração de eventos apresentam realmente maior chance de um indivíduo se candidatar para doação de sangue é importante agregar à análise a população residente em cada região, através de taxas escolhendo para isso uma unidade espacial de análise, que neste estudo foram as áreas de abrangência dos centros de saúde.

O presente estudo mostrou também a importância de se trabalhar com dados de forma desagregada (área de abrangência em vez de, por exemplo, as nove regionais administrativas do município), permitindo assim a localização espacial em pequenas unidades, possibilitando evidenciar desigualdades acentuadas entre as diversas regiões da cidade 20 .

O aumento das taxas Bayesianas empíricas das áreas de abrangência ao norte do município pode estar associado a diversos fatores econômicos e sociais. Dentre eles a violência crescente nesta região 17,18,19, atraindo candidatos de reposição, e a reestruturação e expansão dos meios de transporte da capital mineira nos últimos anos (Empresa de Transporte e Trânsito de Belo Horizonte. http://www.bhtrans.pbh.gov.br, acessado em 15/Jul/2005) - o que facilitou o acesso dos residentes desta área à região central e conseqüentemente ao Hemocentro de Belo Horizonte.

Potencialmente, uma ação conjunta entre a Fundação Hemominas, secretarias municipal e estadual de saúde e comunidade (incluindo suas associações e entidades filantrópicas), pode permitir a implementação de campanhas contínuas e direcionadas aos potenciais doadores - em es- 
pecial em áreas de abrangência com baixas taxas Bayesianas empíricas. Estas ações podem ser implementadas, por exemplo, através das equipes do Programa Saúde da Família (PSF), e podem também auxiliar na elucidação das motivações que resultam em baixas taxas Bayesianas empíricas. Ressalta-se que a estrutura do PSF já se encontra consolidada - inclusive geograficamente - no município e que estas equipes contam com médicos, enfermeiros, auxiliares de enfermagem e agentes comunitários, podendo, todos estes, sensibilizar e informar sobre a importância da doação de sangue e o trabalho da Fundação Hemominas.

As áreas de abrangência localizadas na região mais ao sul do município apresentaram baixa concentração de candidatos. Porém um ponto importante deve ser abordado: este estudo, como citado anteriormente, refere-se a candidatos do Hemocentro de Belo Horizonte localizado na região central do município. Esta baixa concentração nos permite levantar a hipótese de que indivíduos residentes na região sul tendem a se candidatar à doação no posto de coleta instalado no Hospital Júlia Kubistschek (localizado no extremo sul do município e responsável pela coleta de $10 \%$ do sangue), não sendo necessário o deslocamento ao centro do município.

Neste estudo, ao calcularmos as taxas Bayesianas empíricas das áreas de abrangência utilizamos os dados referentes ao Censo Demográfico de 2000. Portanto algumas áreas de abrangência podem ter estes valores subestimados ou superestimados, uma vez que é provável que as áreas de abrangência tenham sofrido alterações do tamanho populacional neste intervalo de dez anos.
Optou-se por trabalhar com a base populacional fixa e referente ao ano de 2000 por serem estimativas seguras e temporalmente intermediárias a 1994 e 2004. Ressalta-se que não existem correspondências entre os setores censitários do Município de Belo Horizonte para os censos de 1991 (1.999 setores censitários) e 2000 (2.563 setores censitários), muitos dos setores de 1991 foram divididos ou agregados. A taxa de crescimento da população de Belo Horizonte foi de 1,9 entre 1994 e 2000, e de 1,2 entre 2000 a 2004 (Departamento de Informática do SUS. http://tabnet.datasus. gov.br/cgi/deftohtm.exe?ibge/cnv/popmg.htm, acessado em 15/Nov/2005), sendo esta variação heterogênea entre as diversas áreas de abrangência que compõem o município, podendo algumas, inclusive, terem tamanho populacional inferior em período subseqüente.

As perdas por georreferenciamento em torno de $13 \%$ revelam a fragilidade da informação de endereços no cadastro dos candidatos à doação de sangue. Mais que a perda na análise espacial, esta fragilidade reflete dificuldade rotineira no serviço para o envio, através dos endereços autofornecidos, dos resultados dos exames realizados no sangue coletado 11 e de correspondências de divulgação e programas de retorno e fidelização aos doadores.

Neste sentido, a implementação de um sistema rotineiro de verificação consistente de endereços no momento do cadastro dos candidatos auxiliará no monitoramento do padrão espacial e na garantia de recebimento das correspondências enviadas pela Fundação Hemominas aos doadores de sangue. 


\section{Resumo}

Este estudo teve como objetivo descrever a distribuição espacial e temporal dos candidatos à doação de sangue, do Município de Belo Horizonte, Minas Gerais, Brasil, que se apresentaram no hemocentro da Fundação Hemominas, nos anos de 1994 e 2004. Comparou no espaço e no espaço-tempo as características idade, sexo e aptidão clínica destes candidatos. As informações de 1994, obtidas de estudo transversal, e as de 2004, amostrados entre os candidatos à doação durante o ano. As amostras foram georreferenciadas através dos endereços de residência. As técnicas espaciais utilizadas foram: mapas Kernel, mapas temáticos de taxas Bayesianas empíricas e de taxas brutas e o índice de Moran global. Os resultados indicaram que a distribuição espacial de candidatos não foi aleatória. Os mapas Kernel auxiliaram na detecção de locais com maior ou menor concentração de candidatos. Os mapas temáticos descreveram a concentração de candidatos relativos à população para as diferentes categorias analisadas. Estes resultados ajudam na detecção de locais para ações direcionadas à captação de candidatos à doação bem como locais com necessidades de campanhas a públicos específicos.

Doadores de Sangue; Análise Espacial; Serviço de Hemoterapia

\section{Colaboradores}

C. Caram participou da concepção e projeto, análise e interpretação dos dados, redação do artigo e aprovação final da versão a ser publicada. M. S. Monteiro-deCastro, C. D. L. Oliveira e M. C. M. Almeida colaboraram na análise e interpretação dos dados. W. T. Caiaffa e F A. Proietti contribuíram na concepção e projeto, revisão crítica do conteúdo intelectual e aprovação final da versão a ser publicada. A. B. F. C Proietti participou da redação do artigo, revisão crítica do conteúdo intelectual e aprovação final da versão a ser publicada. S. Brener colaborou na concepção e projeto e do artigo.

\section{Agradecimentos}

Este trabalho foi parcialmente financiado pelo Conselho Nacional de Desenvolvimento Científico e Tecnológico (CNPq), Fundação de Amparo à Pesquisa do Estado de Minas Gerais (FAPEMIG) e Fundação Hemominas.

\section{Referências}

1. Ministério da Saúde. Campanhas de doação de sangue entram no calendário oficial. Saúde, Brasil 2004; (97):6

2. Oliveira CDL, Loureiro F, Bastos MR, Proietti FA, Carneiro-Proietti AB. Blood donor deferral in Minas Gerais State, Brazil: blood centers as sentinels of urban population health. Transfusion 2009; 49:851-7.

3. Custer B, Johnson ES, Sullivan SD, Hazlet TK, Ramsey SD, Hirschler NV, et al. Qualifying losses to the donated blood supply due to donor deferral and miscollection. Transfusion 2004; 44:1417-26.

4. Verchenko SB, Sakurai E, Caiaffa WT, Proietti FA Fatores associados à aptidão clínica para a doação de sangue - determinantes demográficos e sócioeconômicos. Rev Bras Hematol Hemoter 2008; 30:108-13.

5. Czeresnia D, Ribeiro AM. O conceito de espaço em epidemiologia: uma interpretação histórica e epistemológica. Cad Saúde Pública 2000; 16:595-605.
6. Givisiez AGN, Vertchenko SB, Rodrigues RN, Fígoli MGB, Caiaffa WT, Proietti FA. Aspectos sócio-econômicos, demográficos e culturais da doação de sangue em Minas Gerais e estudo do perfil epidemiológico, das infecções passíveis de transmissão através das transfusões em doadores de sangue e população de base em Minas Gerais. Relatório técnico. Belo Horizonte: Faculdade de Medicina, Universidade Federal de Minas Gerais; 1998.

7. Bailey TC, Gatrell AC. Interactive spatial data analysis. Essex: Longman Scientific \& Technical; 1995.

8. Câmara G, Monteiro AMV, Druck S, Carvalho MS. Análise espacial de dados geográficos. Brasília: Empresa Brasileira de Pesquisa Agropecuária; 2004.

9. Assunção RM, Barreto SM, Guerra HL, Sakurai E. Mapas de taxas epidemiológicas: uma abordagem Bayesiana. Cad Saúde Pública 1998; 14:713-23. 
10. Rowlingson B, Diggle PJ. SPLANCS: spatial point pattern analysis code in S-Plus. Comput Geosci 1993; 19:627-55.

11. Monteiro-de-Castro MS, Assunção RM, Proietti FA. Spatial distribution of the human T-lymphotropic virus types I and II (HTLV-I/II) infection among blood donors of Hemominas Foundation, Belo Horizonte, Minas Gerais State, Brazil, 1994-1996. Cad Saúde Pública 2001; 17:1219-30.

12. Almeida CM, Caiaffa WT, Assunção RM, Proietti FA. Spatial vulnerability to dengue in a Brazilian urban area during a 7-year surveillance. J Urban Health 2007; 84:334-45.

13. Santos SM, Barcellos C, Carvalho MS, Flôres R. Detecção de aglomerados espaciais de óbitos por causas violentas em Porto Alegre, Rio Grande do Sul, Brasil, 1996. Cad Saúde Pública 2001; 17:1141-51.

13. Shimakura SE, Carvalho MS, Aerts DRGC, Flores R. Distribuição espacial do risco: modelagem da mortalidade infantil em Porto Alegre, Rio Grande do Sul, Brasil. Cad Saúde Pública 2001; 17:1251-61.

14. Oliveira CL, Assunção RM, Reis IA, Proietti FA. Spatial distribution of human and canine visceral leishmaniasis in Belo Horizonte, Minas Gerais State, Brasil, 1994-1997. Cad Saúde Pública 2001; 17:1231-9
15. Moran J, Barnard S, Jones K, Twigg L. Social capital, place and health: creating, validating and applying small-area indicators in the modelling of health outcomes. London: Health Development Agency, National Institute for Health and Clinical Excellence; 2004.

16. Caiaffa WT, Almeida MCM, Oliveira CL, Friche AAL, Matos SG, Dias MAS, et al. The urban environment from the health perspective: the case of Belo Horizonte, Minas Gerais, Brazil. Cad Saúde Pública 2005; 21:958-67.

17. Beato Filho CC, Assunção RM, Silva BFA, Marinho FC, Reis IA, Almeida MCM. Conglomerados de homicídios e o tráfico de drogas em Belo Horizonte, Minas Gerais, Brasil, de 1995 a 1999. Cad Saúde Pública 2001; 17:1163-71.

18. Matos SG, Proietti FA, Barata RCB. Confiabilidade da informação sobre mortalidade por violência em Belo Horizonte, MG. Rev Saúde Pública 2007; 41:76-84.

19. Malta DC, Almeida MCM, Dias MAS, Merhy EE. A mortalidade infantil em Belo Horizonte, Minas Gerais, Brasil, por área de abrangência dos Centros de Saúde (1994-1996). Cad Saúde Pública 2001; 17:1189-98.

20. Machado-Coelho GL, Assunção R, Mayrink W, Caiaffa WT. American cutaneous leishmaniasis in Southeast Brazil: space-time clustering. Int J Epidemiol 1999; 28:982-9.

Recebido em 29/Out/2008

Versão final reapresentada em 23/Out/2009

Aprovado em 10/Nov/2009 